access to services and transplantation. Prior to developing a protocol for attempted eradication of Bcc, similar to that applied to Pseudomonas aeruginosa, UK CF centres were surveyed to establish what practices are currently employed.

Methods A questionnaire was distributed to CF centre directors and non-returns followed up by email reminder.

Results Responses were obtained from 35 units (73\%). Because of the rarity of new Bcc infection in paediatric centres, only replies from 16 adult centres (representing 3400 patients) have been analysed further. 12 centres, representing 2860 patients, always attempt eradication of newly isolated Bcc. Two additional centres attempt eradication only in the presence of additional indications. Only two units had a formal eradication policy. IV antibiotics were used in all cases, for a median of 2 (range 2-6) weeks, typically comprising combined tobramycin and meropenem with additional therapy consisting of septrin $(n=5)$, ceftazidime (6), and chloramphenicol (2). Nebulised antibiotics (typically tobramycin or meropenem) were also used in 13 of these 14 centres. Five units used additional oral antibiotics, for a median of 7 $(2-12)$ weeks. This most commonly involved minocycline $(n=4)$ and/or septrin $(\mathrm{n}=5)$. Two thirds of adult centres estimated success rate of eradication therapy to be $<50 \%$. In centres where eradication was not routine, factors that dissuaded clinicians were perceived poor success of treatment $(n=5)$, toxicity $(n=3)$, cost $(n=1)$ and lack of experience with this approach $(n=2)$.

Conclusions Attempted eradication of newly acquired Bcc is controversial, involving expensive and potentially toxic therapies with no evidence to guide treatment choice and no published outcome data. Despite this, it is common practice in many UK adult CF centres. Most units do not have a formal eradication policy, adopting a variety of approaches, and estimates of treatment success are pessimistic. Since new acquisition of Bcc is now relatively rare, it is hard for even large units to assess response to therapies. A systematic approach is required to optimise and standardise treatment regimens, and assess outcomes.

\section{P169 CHARACTERISTICS AND SURVIVAL OF PATIENTS WITHOUT CYSTIC FIBROSIS (CF), ISOLATING MUCOID P AERUGINOSA (MPA) IN SPUTUM SAMPLES}

\section{doi:10.1136/thoraxjnl-2011-201054c.169}

R Ahmed, M Denton, I Clifton, D Peckham. Leeds Teaching Hospitals NHS Trust, Leeds, UK

Introduction and Objectives It is well established in patients with CF $\mathrm{m} P A$ indicates chronic infection and is associated with increased morbidity and mortality. Mucoid PA can be isolated in people with non-CF lung disease but to-date no study has systematically characterised these patients. The aim of this study was to characterise and evaluate the significance of mucoid strain in a large non CF population

Methods This study retrospectively examined the demography, clinical diagnoses and survival, of patients without CF with an initial isolate of $\mathrm{mPA}$ from their respiratory samples, between January 2008 and December 2010, in Leeds. Data were obtained from the microbiology and radiology databases, and from the clinical notes. Ethical approval was obtained.

Results A total of 170 patients isolated $\mathrm{mPA}$ in sputum for the first time during the study period. The median (range) age was 73 (22-99) years and $54 \%$ were female. $46 \%$ of patients had samples obtained during an in-patient stay. $21 \%$ of the patients had never seen a respiratory physician. The majority of patients had a diagnosis of bronchiectasis (See Abstract P169 figure 1). A third of patents had isolated non-mucoid $P A$ prior to isolating $\mathrm{mPA}$. $62 \%$ of the patients had a subsequent respiratory sample analysed and the majority continued to grow $\mathrm{mPA}$. 24 patients also grew Methicillinresistant Staphylococcus aureus (MRSA) from their sputum. The median age (range) of death was 78 (40-95) years. The cumulative survival was $50 \%$ at 3 years after isolating $\mathrm{mPA}$. Factors associated with increased mortality included co-isolation of MRSA, no previous respiratory review, isolating $\mathrm{m} P A$ during in-patient care and absence of bronchiectasis $(p<0.05)$.

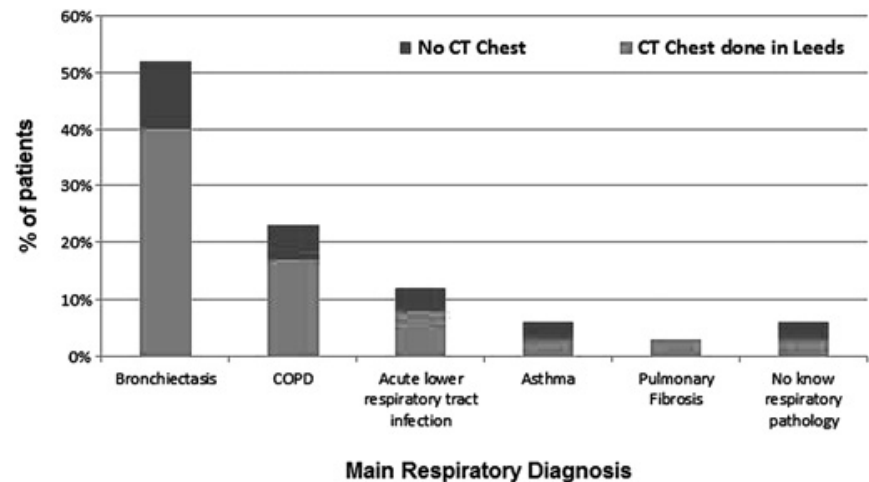

Abstract P169 Figure 1 Diagnosis of patients isolating $\mathrm{mPA}$.

Conclusions Mucoid $P$ aeruginosa isolated from the sputum of patients without CF may persist and is associated with high mortality. The underlying respiratory diagnosis of patients isolating $\mathrm{m} P A$ includes a range of respiratory diseases. There may be an opportunity to eradicated $P$ aeruginosa in patients who grow nonmucoid $P A$ before the emergence of $\mathrm{m} P A$.

\section{P170 A COST-EFFECTIVENESS ANALYSIS OF RESPIRATORY SYNCYTIAL VIRUS (RSV) PROPHYLAXIS IN INFANTS IN THE UK}

doi:10.1136/thoraxinl-2011-201054c.170

${ }^{1}$ A Bentley, ${ }^{2}$ Filipovic, ${ }^{2} \mathrm{~K}$ Gooch, ${ }^{2} \mathrm{~K}$ Buesch. ${ }^{1}$ Abacus International, Bicester, UK; ${ }^{2}$ Abbott Laboratories, Solna, Sweden

Background and Aim RSV is the most common cause of lower respiratory tract infection in young children and may result in hospitalisations, long-term sequelae, such as recurrent wheeze and/or asthma, and possibly death. Palivizumab is a prophylaxis against severe RSV infection and has been shown to reduce RSV-related hospitalisations, and provides a preventative option for high-risk infants. The aim of this study is to demonstrate the cost-effectiveness of RSV prophylaxis compared to no prophylaxis in the following high-risk infants groups: infants with chronic lung disease (CLD) and premature babies $(<29$, 29-32 and 33-35 weeks gestational age (wGA)).

Methods A decision-tree model was developed to reflect the clinical pathway of infants at high-risk of severe RSV infection. Baseline risk of RSV-hospitalisations and efficacy data were taken from palivizumab clinical trials and published meta-analysis. Cost data were obtained from national databases and published literature using an NHS perspective. The main outcome was presented as the incremental costs per Quality-Adjusted Life-Year (OALY) gained (ICER). The base-case analysis considered a lifetime horizon to capture the impact of long-term morbidity and mortality associated with RSV hospitalisations.

Results Prophylaxis against severe RSV infection resulted in ICERs of $£ 19168$, $£ 18174$ \& $£ 1185$ per OALY for high-risk infants with, CLD and the premature infant groups, $<29$ wGA and 29-32 wGA respectively compared to no prophylaxis. All results are below the accepted NICE threshold of $£ 30000$ /OALY thus demonstrating cost-effectiveness. The baseline ICER for the 33-35 wGA subgroup was above this threshold however, sensitivity analysis considering risk-factors in this subgroup showed that an increase in baseline risk of hospitalisation, from $7.2 \%$ to $11.24 \%$, led to palivizumab becoming a cost-effective option. 